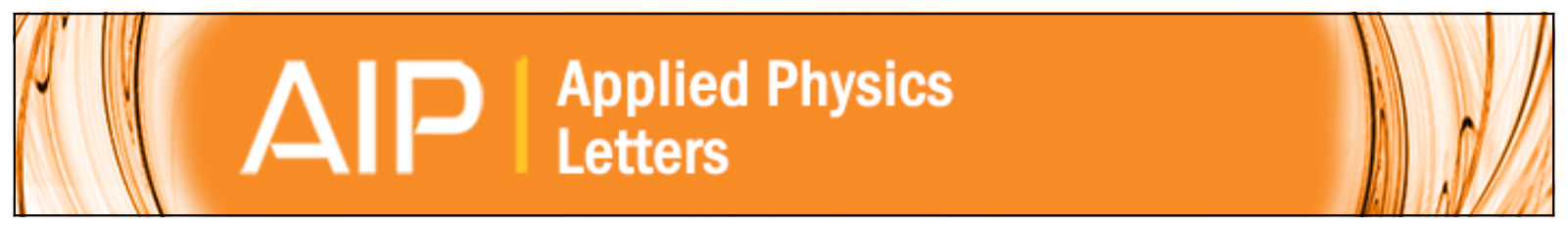

Oligomer-based organic distributed feedback lasers by room-temperature nanoimprint lithography

Dario Pisignano, Luana Persano, Paolo Visconti, Roberto Cingolani, Giuseppe Gigli, Giovanna Barbarella, and Laura Favaretto

Citation: Applied Physics Letters 83, 2545 (2003); doi: 10.1063/1.1613362

View online: http://dx.doi.org/10.1063/1.1613362

View Table of Contents: http://scitation.aip.org/content/aip/journal/apl/83/13?ver=pdfcov

Published by the AIP Publishing

Articles you may be interested in

Highly photostable organic distributed feedback laser emitting at $573 \mathrm{~nm}$

Appl. Phys. Lett. 97, 171104 (2010); 10.1063/1.3506500

Evaluation of nanoimprint lithography as a fabrication process of phase-shifted diffraction gratings of distributed feedback laser diodes

J. Vac. Sci. Technol. B 27, 2776 (2009); 10.1116/1.3244631

Simple fabrication technique of distributed-feedback polymer laser by direct photonanoimprint lithography Appl. Phys. Lett. 92, 243306 (2008); 10.1063/1.2945632

Polymeric distributed feedback lasers by room-temperature nanoimprint lithography

Appl. Phys. Lett. 89, 131109 (2006); 10.1063/1.2357116

Laser action from two-dimensional distributed feedback in photonic crystals

Appl. Phys. Lett. 74, 7 (1999); 10.1063/1.123116

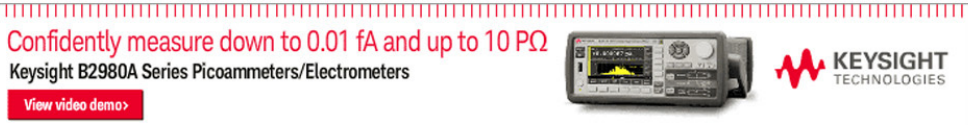




\title{
Oligomer-based organic distributed feedback lasers by room-temperature nanoimprint lithography
}

\author{
Dario Pisignano, ${ }^{\text {a) }}$ Luana Persano, Paolo Visconti, Roberto Cingolani, and Giuseppe Gigli \\ NNL, National Nanotechnology Laboratory of Istituto Nazionale di Fisica della Materia (INFM), c/o \\ Dipartimento di Ingegneria dell'Innovazione, Università di Lecce, via Arnesano, I-73100 Lecce, Italy \\ Giovanna Barbarella and Laura Favaretto \\ Consiglio Nazionale delle Ricerche (CNR), ISOF, Area della Ricerca di Bologna, via Gobetti 101, \\ I-40129 Bologna, Italy
}

(Received 14 April 2003; accepted 28 July 2003)

\begin{abstract}
Room-temperature nanoimprint lithography in air is used in order to pattern a nonthermoplastic, low-molar-mass thiophene-based pentamer with excellent gain properties. No degradation of the luminescence efficiency of the active medium was observed after patterning. In this way, we fabricated single-mode emission distributed feedback lasers having a threshold excitation fluence of $140 \mu \mathrm{J} / \mathrm{cm}^{2}$. The lasing line is peaked at $637 \mathrm{~nm}$ and exhibits a linewidth of less than $0.7 \mathrm{~nm}$ and a well-behaved input-output characteristic in the whole range of pump fluences. These results demonstrate room-temperature nanoimprint lithography as powerful and straightforward fabrication technique for oligomer-based nanostructured optoelectronic devices. (c) 2003 American Institute of Physics. [DOI: 10.1063/1.1613362]
\end{abstract}

Nanoimprint lithography (NIL) ${ }^{1}$ exploits the glass transition of polymers to transfer with very high fidelity master patterns realized by conventional lithographic techniques. It employs a rigid mold to directly pattern micro- and nanostructures into target polymers, ${ }^{1}$ with no need of exposure to radiation, development, and etching processes, offering the large-area and low-cost operation typical of mechanical lithographies. ${ }^{2}$ Besides other results, the realization by imprinting of direct three-dimensional patterning, ${ }^{3}$ nanoscale field effect transistors, ${ }^{4}$ metal-semiconductor-metal photodetectors, ${ }^{5}$ and sub-10 nm structures have been demonstrated. $^{6}$

During the imprinting process, the mold is placed onto a cast film of the target polymer. The overall system is then driven at a temperature considerably higher than the glass transition temperature, $T_{g}$, of the target. Once at the liquid state, the target material takes the shape of the pattern in the master. The subsequent cooling of the system below $T_{g}$ freezes the pattern on the target surface, thus providing a negative copy of the master. Such a thermal cycle is an intrinsic problem of conventional NIL, as it requires a thermoplastic behavior of the target compound which can be hardly achieved in small molecules, thus preventing their use as directly printed active materials. Nevertheless, in the last years many low-molar-mass compounds have been found to exhibit excellent luminescence and optical gain performances, ${ }^{7}$ thus raising the interest in patterning methods able to directly pattern them.

Moreover, since heating the samples can cause the degradation of the optical and electrical properties of the organic materials NIL has to be performed under vacuum to pattern light-emitting molecules, ${ }^{8}$ and room-temperature processes have been proposed for conductive polymers, including

\footnotetext{
a) Also at: Dipartimento di Fisica, Università di Lecce, via Arnesano, I-73100 Lecce, Italy; electronic mail: dario.pisignano@unile.it
}

imprinting $^{9}$ and micromolding in capillaries. ${ }^{10}$

In this letter, room-temperature NIL is employed in order to pattern a low-molar-mass, nonthermoplastic gaining molecule, namely the thiophene-based oligomer $3,3^{\prime}, 4^{\prime \prime \prime}, 3^{\prime \prime \prime}$ tetracyclehexyl-3", $4^{\prime \prime}$ - dihexyl-2, $2^{\prime}: 5^{\prime}, 2^{\prime \prime}: 5^{\prime \prime}, 2^{\prime \prime \prime}: 5^{\prime \prime \prime}, 2^{\prime \prime \prime \prime}$ :quinquethiophene- $1^{\prime \prime}, 1^{\prime \prime}$-dioxide (T5oCx). As a demonstrator, we fabricate a directly printed distributed feedback (DFB) laser, exhibiting single-mode emission at about $640 \mathrm{~nm}$, with a full width at half maximum (FWHM) of less than $0.7 \mathrm{~nm}$ and a pump threshold of $140 \mu \mathrm{J} / \mathrm{cm}^{2}$. The lithography process allows a highly faithful pattern transfer without reducing the emission yield, thus opening the way to the one-step realization of oligomer-based optoelectronic devices.

Our lithography process is schematized in Fig. 1(a). T5oCx [whose molecular structure is displayed in Fig. 1(b)] films were cast onto Corning glass substrates, from chloroform solutions (concentration $\cong 7 \times 10^{-2} \mathrm{M}$, spin-coating speed $1600 \mathrm{rpm}$ ). Master patterns with a period, $\Lambda=600 \mathrm{~nm}$, and an area of $3 \mathrm{~mm} \times 8 \mathrm{~mm}$ were first fabricated on silicon by electron beam lithography with a Leica LION LVI system working at acceleration energy of $2.5 \mathrm{keV}$, and subsequent lift-off and conventional reactive ion etching by a $\mathrm{CF}_{4} / \mathrm{Ar}$ mixture chosen in order to minimize the plasma-induced polymer deposition (Fig. 2). The master was then directly used as a stamp for nanoimprint, employing a precision manual press (PW100 P/O/Weber, Germany) at room temperature, in air, with an applied force of about $5 \mathrm{kN}$. No antisticking layer was needed in the imprinting process. After separating the stamp from the patterned sample, the obtained DFB device was excited by the third harmonic $(\lambda=355 \mathrm{~nm})$ of a 3 ns $Q$-switched Nd:yttrium-aluminum-garnet laser (Spectra-Physics, repetition rate of $10 \mathrm{~Hz}$ ). The excitation beam was focused on the sample by a cylindrical lens, thus providing a rectangular excitation stripe of width $\cong 500 \mu \mathrm{m}$. The DFB emission at room temperature was dispersed by a monochromator and detected by a Si charge coupled device, 

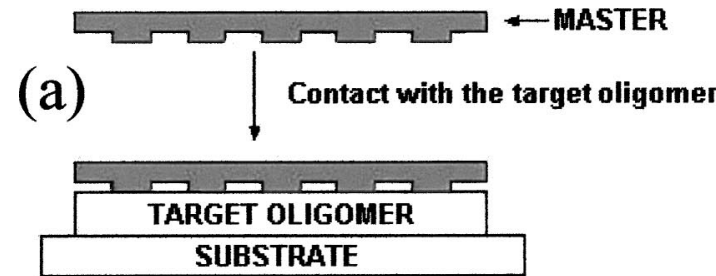

Applying pressure (No heating)
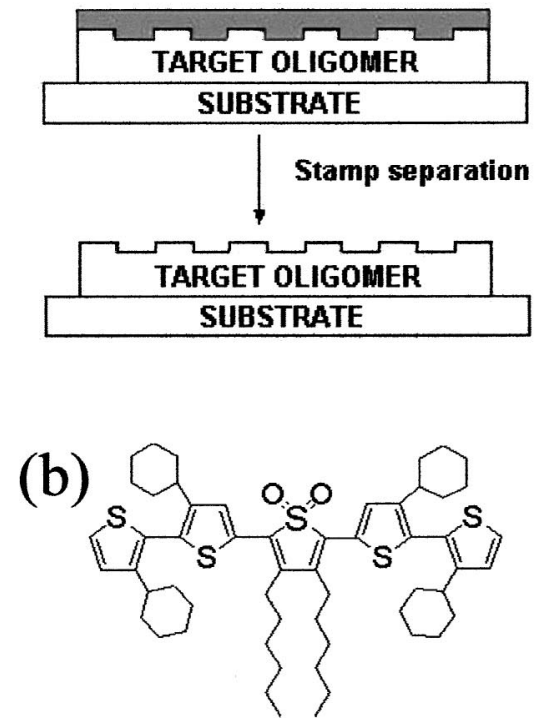

FIG. 1. (a) Schematic diagram of the process of room-temperature nanoimprint lithography (features not in scale). (b) Molecular structure of T5oCx.

providing a spectral resolution of about $0.1 \mathrm{~nm}$. The photoluminescence (PL) efficiency, $\eta_{\mathrm{PL}}$, before and after patterning, was determined by an integrating sphere. The optical measurements were carried out under vacuum $\left(10^{-6}\right.$ mbar $)$ to inhibit photo-oxidation.

$\mathrm{T} 5 \mathrm{oCx}$ exhibits excellent film-forming processing and solubility, good electroluminescence, ${ }^{11}$ amplified spontaneous emission, and lasing performances among the best reported for organic compounds. ${ }^{12}$ However, like many other low-molar-mass molecules, it cannot be directly imprinted by conventional NIL or soft molding techniques ${ }^{13}$ due to its poor thermoplastic properties. In this case, one has to use room-temperature nanoimprint, which exploits only the ap-

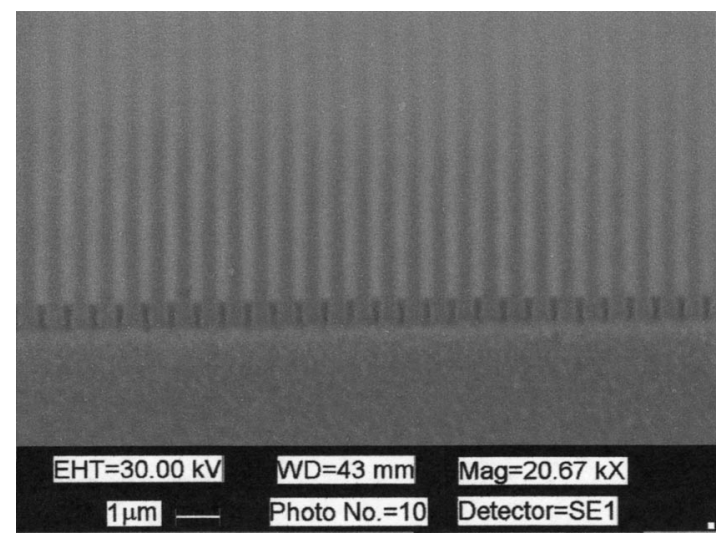

FIG. 2. Cross section of the realized master gratings imaged by scanning electron microscopy The features are more than $500 \mathrm{~nm}$ high (aspect ratio larger than 1.6).

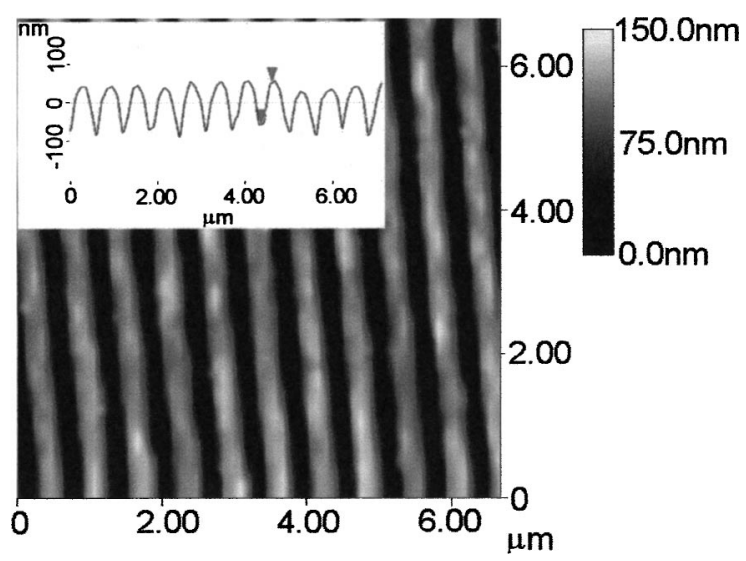

FIG. 3. AFM two-dimensional view of the patterned $\mathrm{T} 5 \mathrm{oCx}$ grating. Inset: AFM cross section of the final grating onto T50Cx. The height of the obtained features is more than $110 \mathrm{~nm}$.

plied pressure to transfer the pattern into the target organic film, thus avoiding the drawbacks due to the thermal cycle needed in other embossing processes. We underline that, differently from previously reported room-temperature nanoimprinting experiments, ${ }^{14}$ our results demonstrate the one-step realization of optoelectronic devices with nonthermoplastic organic compounds, which cannot be imprinted by conventional hot embossing. Moreover, room-temperature NIL does not require vacuum or controlled atmosphere to avoid the degradation of the optical properties of the organic semiconductor. The obtained $\mathrm{T} 5 \mathrm{oCx}$ grating, imaged by atomic force microscopy (AFM), is shown in Fig. 3. The measured photoluminescence efficiency, $\eta_{\mathrm{PL}}$, of T5oCx films before and after room-temperature imprint was found to be constant around the value of $18 \%$, confirming that no degradation of the luminescence performances of the oligomer is caused by the lithography process.

The patterned T5oCx surface was then used as the corrugated interface of a printed, optically pumped DFB device. ${ }^{15}$ In organic DFBs, the gain medium is usually deposited on previously patterned, optically inert substrates, such as silica or transparent polymers. ${ }^{16-22}$ After such a process, the upper surface of the deposited gain film is partially planarized, ${ }^{16,17}$ so that the interface between the active medium and air is quite smeared out, strongly affecting the DFB emission. To date, methods to fabricate organic DFB exploited as active medium conjugated polymers, ${ }^{19,20}$ and blends incorporating commercial dyes. ${ }^{17,18,21-24}$ Our approach, instead, guarantees an oligomer-based DFB whose active medium-air interface is quite sharp and can be precisely controlled by the nanoimprinting.

The lasing emission from our printed DFB is peaked within the gain spectral region of $\mathrm{T} 5 \mathrm{oCx}$, which we found to be extended between 580 and $660 \mathrm{~nm}$. The single-mode emission is peaked at $\lambda_{m}=637 \mathrm{~nm}$, with a FWHM of less than $0.7 \mathrm{~nm}$ and a threshold excitation fluence of $140 \mu \mathrm{J} / \mathrm{cm}^{2}$ (Fig. 4). According to the well known equation, $m \lambda_{m}$ $=2 n_{\text {eff }} \Lambda$, where $m$ indicates the diffraction order onto the DFB grating and $n_{\text {eff }}$ is the effective refractive index of the propagating mode, the device works at the third order of diffraction, with $n_{\text {eff }}$ of the propagating mode as high as 1.6. Finally, the DFB peak emission grows linearly with the excitation, as expected for laser action (inset of Fig. 4). 


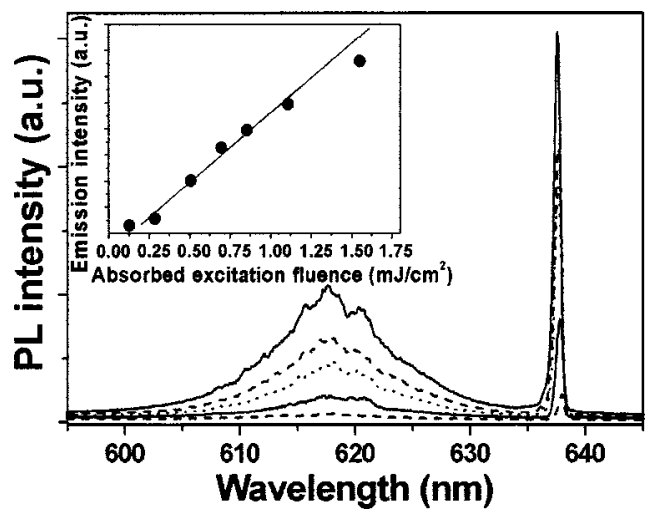

FIG. 4. DFB emission spectra collected at different absorbed excitation fluences (from bottom to top: $0.3,0.5,0.9,1.1,1.6 \mathrm{~mJ} \mathrm{~cm}^{-2}$ ). Inset: DFB peak emission intensity vs fluence of the absorbed excitation beam. The solid line is a linear fit to the experimental data.

In conclusion, we demonstrate a high-quality, oligomerbased organic DFB device realized by one-step nanoimprinting at room temperature in air. This patterning technique extends the excellent resolution, large-area operation and low-cost of conventional NIL to another, very wide and useful class of molecules, namely low-molar-mass compounds with poor thermoplastic behavior, thus allowing a full exploitation of the chemical flexibility characteristic of mechanical lithographies. Further work is currently in progress in our laboratory to assess the ultimate resolution and the applicability of room-temperature nanoimprint to other low-molarmass, high gain organic materials.

${ }^{1}$ S. Y. Chou, P. R. Krauss, and P. J. Renstrom, Appl. Phys. Lett. 67, 3114 (1995); Science 272, 85 (1996).

${ }^{2}$ Y. Xia and G. M. Whitesides, Angew. Chem., Int. Ed. 37, 550 (1998).

${ }^{3}$ M. Li, L. Chen, and S. Y. Chou, Appl. Phys. Lett. 78, 3322 (2001).
${ }^{4}$ L. Guo, P. R. Krauss, and S. Y. Chou, Appl. Phys. Lett. 71, 1881 (1997).

${ }^{5}$ Z. Yu, S. J. Schablitsky, and S. Y. Chou, Appl. Phys. Lett. 74, 2381 (1999).

${ }^{6}$ S. Y. Chou, P. R. Krauss, W. Zhang, L. Guo, and L. Zhuang, J. Vac. Sci. Technol. B 15, 2897 (1997).

${ }^{7}$ See D. Pisignano, M. Anni, G. Gigli, R. Cingolani, M. Zavelani-Rossi, G. Lanzani, G. Barbarella, and L. Favaretto, Appl. Phys. Lett. 81, 3534 (2002), and references therein.

${ }^{8}$ J. Wang, X. Sun, L. Chen, and S. Y. Chou, Appl. Phys. Lett. 75, 2767 (1999).

${ }^{9}$ M. Behl, J. Seekamp, S. Zankovych, C. M. Sotomayor Torres, R. Zentel, and J. Ahopelto, Adv. Mater. (Weinheim, Ger.) 14, 588 (2002).

${ }^{10}$ W. S. Beh, I. T. Kim, D. Qin, Y. Xia, and G. M. Whitesides, Adv. Mater. (Weinheim, Ger.) 11, 1038 (1999).

${ }^{11}$ G. Gigli, O. Inganas, M. Anni, M. De Vittorio, R. Cingolani, G. Barbarella, and L. Favaretto, Appl. Phys. Lett. 78, 1493 (2001).

${ }^{12}$ D. Pisignano, M. Anni, G. Gigli, R. Cingolani, M. Zavelani-Rossi, G. Lanzani, G. Barbarella, and L. Favaretto, Appl. Phys. Lett. 81, 3534 (2002).

${ }^{13}$ K. Y. Suh, Y. S. Kim, and H. H. Lee, Adv. Mater. (Weinheim, Ger.) 13, 1386 (2001).

${ }^{14}$ D.-Y. Khang, H. Yoon, and H. H. Lee, Adv. Mater. (Weinheim, Ger.) 13, 749 (2001).

${ }^{15}$ H. Kogelnik and C. V. Shank, Appl. Phys. Lett. 18, 152 (1971).

${ }^{16}$ G. A. Turnbull, P. Andrews, M. J. Jory, W. L. Barnes, and I. D. W. Samuel, Phys. Rev. B 64, 125122 (2001).

${ }^{17}$ M. Bergreen, A. Dodabalapur, and R. E. Slusher, Appl. Phys. Lett. 71, 2230 (1997).

${ }^{18}$ M. Bergreen, A. Dodabalapur, R. E. Slusher, A. Timko, and O. Nalamasu, Appl. Phys. Lett. 72, 410 (1998).

${ }^{19}$ N. Moll, R. F. Mahrt, C. Bauer, H. Giessen, B. Schnabel, E. B. Kley, and U. Scherf, Appl. Phys. Lett. 80, 734 (2002).

${ }^{20}$ S. Riechel, C. Kallinger, U. Lemmer, J. Feldmann, A. Gombert, V. Wittwer, and U. Scherf, Appl. Phys. Lett. 77, 2310 (2000).

${ }^{21}$ J. A. Rogers, M. Meier, and A. Dodabalapur, Appl. Phys. Lett. 73, 1766 (1998).

${ }^{22}$ J. A. Rogers, M. Meier, A. Dodabalapur, E. J. Laskowski, and M. A. Cappuzzo, Appl. Phys. Lett. 74, 3257 (1999).

${ }^{23}$ B. J. Scott, G. Wirnsberger, M. D. McGehee, B. F. Chmelka, and G. D. Stucky, Adv. Mater. (Weinheim, Ger.) 13, 1231 (2001).

${ }^{24}$ S. Riechel, U. Lemmer, J. Feldmann, S. Berleb, A. G. Mückl, W. Brütting, A. Gombert, and V. Wittwer, Opt. Lett. 26, 593 (2001). 